\title{
Control of Lower Limb Rehabilitation Robot Based on Fuzzy PID
}

\author{
Wenyu Huang, Haibo $\mathrm{Xu}^{*}$, Xing Fan and Yufeng Lin \\ School of Mechanical Engineering, Xi'an Jiaotong University, Xi'an 710049, China \\ ${ }^{*}$ Corresponding author
}

\begin{abstract}
For the problem of speed control of passive training in lower limb rehabilitation robot, a control strategy based on fuzzy PID is proposed. Firstly, the trajectory curve of the position was derived and the fuzzy controller was designed. The kinematics model and the simulation model of the controlled object were established based on Matlab/Simulink and Adams. The simulation results showed that the proposed control strategy was feasible and more classic. The PID control has the advantages of small overshoot, fast response, and high steady-state accuracy. It can better track the planned motion curve and meet the system control performance requirements.
\end{abstract}

Keywords - lower limb rehabilitation robot; speed control; fuzzy PID; Matlab-Adams co-simulation

\section{INTRODUCTION}

Medical research has shown that patients with limb dyskinesia caused by nerve damage and healthy limbs can help them recover their athletic ability through scientific and effective exercise rehabilitation training [1]. In recent years, with the rapid development of robotics, many scholars combine intelligent robot technology with rehabilitation medicine, and use the characteristics of high robot motion precision, accurate data state acquisition, strong interaction, intelligent automatic to assist or replace the rehabilitation doctor to complete the sports therapy. Rehabilitation robots came into being.

At present, rehabilitation robots still have shortcomings such as low intelligence and poor rehabilitation. In view of this situation, medically analyzing the movement relationship between the normal limb and the affected limb, the preset lower limb training trajectory, the effect of lower limb movement controlled by the lower limb rehabilitation robot is achieved.

In this paper, aiming at the higher requirements of the rehabilitation training mode for the accuracy of the motion track following control, the fuzzy PID control algorithm is used to realize the speed control of the lower limb rehabilitation robot. Through the joint simulation model of control system and dynamics system established by Matlab and Adams, the speed control effect of fuzzy PID algorithm is simulated and analyzed.

\section{LOWER LIMB REHABILITATION STRATEGY}

\section{A. Passive Rehabilitation Strategy}

The overall structure of the pedal-type lower limb rehabilitation robot described in this paper is shown in Figure I. It mainly includes drive and transmission structure, rotating crank, limb protection structure, fixed frame and so on.

In the early stage of rehabilitation training, the patient's limb muscle strength is very weak, joint resistance is large or muscle stiffness still exists. In the physical therapy of traditional rehabilitation physicians, the use of human hands to drive the limbs to relax the muscles of the affected limbs and reduce the joint activity resistance. When the lower limb rehabilitation robot is training, the motor is used to drive the affected limbs for rehabilitation training. In order to ensure that the training has a positive effect on the affected limb and avoid the negative influence of the wrong movement mode on the limb movement, the rehabilitation training is carried out in accordance with the trend of the normal person pedaling speed.

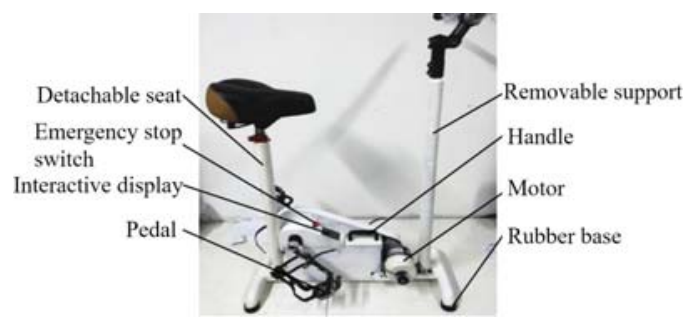

FIGURE I. LOWER LIMB REHABILITATION ROBOT PHYSICAL MAP

\section{B. Position Velocity Trajectory Curve}

In the case where the driving motor does not provide the main power, the relationship between the crank angle position and the crank angular velocity of the volunteer $\mathrm{A}$ and the volunteer B during normal treadmill movement is collected. In order to observe the positional regularity of the angular velocity data, the multi-cycle curves are stacked by position and averaged at corresponding angular positions to form a treadmill position-angular velocity curve, as shown in Figure II. It can be seen from the figure that the crank angular velocity changes periodically with the crank position during the treadmill movement. The pedaling speed of the left and right limbs is slightly different but similar. The relative minimum angular velocity point is near the transition position of the left and right 
limbs, and the movement is not violently shaken, which is basically stable.

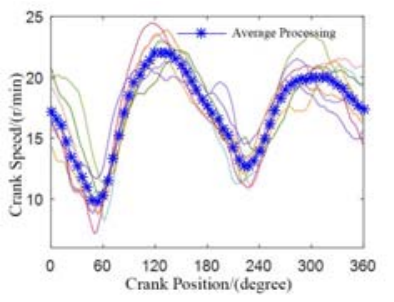

(a) volunteer $\mathrm{A}$

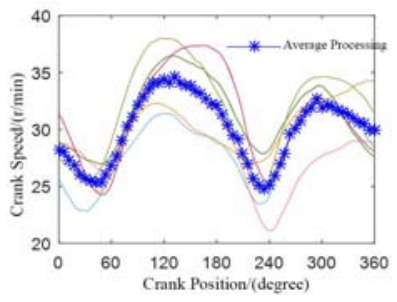

(b) volunteer B
FIGURE II. NORMAL PERSON TREADMILL POSITION - SPEED TRAJECTORY DIAGRAM

Observing the position-speed trajectory map of Figure II, it is found that there are some deviations between the left and right limb movement trajectories of normal people in the treadmill exercise, but the overall motion law is similar. The speed will decrease near the critical point of the pedaling, and the overall position-speed trajectory is approximately sinusoidal. Therefore, the law of motion trajectory when setting passive training is as shown in equation (1) [2].

$$
\omega\left(\theta_{c r}\right)=H+A \sin \left(2 \pi \times \frac{\theta_{c r}+135-\theta_{c}}{180}\right)
$$

Among them: $\omega\left(\theta_{c r}\right)$-the target speed at the crank position $\theta_{c r} /(\mathrm{r} / \mathrm{min}) ; H$-the average speed of the treadmill movement/(r/min); $A$-the sinusoidal speed track amplitude/(r/min); $\theta_{c}$-Treadmill critical point angular position / (degree).

As can be seen from Figure II, the larger the average speed $\mathrm{H}$, the less the speed reduction at the critical point of the treadmill, and the smaller A is. This is because when the speed is faster, the lower limb inertia can help the body to quickly cross the critical point and enter the next pedaling stage.

\section{SIMULATION ANALYSIS}

\section{A. Adams Dynamics Model}

The Adams dynamic model incorporates a three-dimensional model of the lower limb rehabilitation robot and a human body model. Individual human inertia and other parameters can be determined according to the "Chinese adult human body size (GB/T 10000-1988)" using the binary regression equation, the parameters of the human lower limb simulation model of $172 \mathrm{~cm}$ in height and $62 \mathrm{~kg}$ in body weight were determined[3]. In the co-simulation, the Adams input parameters are the crankshaft rotation torque and the human body lower limb output main force. The output is the crankshaft angular velocity (unit: degree per second) and the shaft position angle (unit: degree).

\section{B. Fuzzy PID Controller Design}

Fuzzy PID is based on the PID algorithm. By calculating the current system error value $e$ and the error change rate $e c$, using fuzzy rules to perform fuzzy reasoning, the fuzzy matrix table is used to query the PID parameters of the PID parameters. Fuzzy PID is based on the PID algorithm. By calculating the current system error value $e$ and the error change rate $e c$, using fuzzy rules to perform fuzzy reasoning, the fuzzy matrix table is used to query the PID parameters of the PID parameters[4].

The fuzzy PID uses the error value $e$ and the error change rate $e c$ as the fuzzy controller input, and $\Delta K_{p} 、 \Delta K_{d} 、 \Delta K_{i}$ as the fuzzy controller output, which is divided into seven fuzzy subsets according to the experience. Fuzzy PID uses Mamdani fuzzy inference method, and the input and output response is obtained by MATLAB calculation using the minimum operation rule and the center of gravity method to deblur as shown in Figure III. The motor model is packaged and added to the Matlab simulation model with the established fuzzy controller and PID controller to obtain the fuzzy PID simulation model.
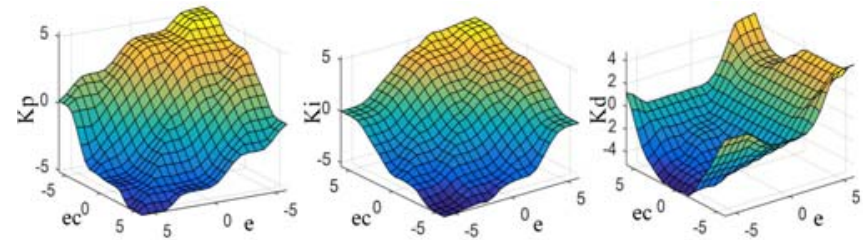

FIGURE III. FUZZY PID INPUT AND OUTPUT RESPONSE DIAGRAM

Through multiple debugging and analysis of the variation range of $e$ and $e c$, the proportional quantization factors are $K e=0.5$ and $K e c=0.5$. And the output ratio quantization factors are $K \_K p=0.1, K \_K i=0.1, K \_K d=0.5$, and the initial value is set to $40,0.8,900$. The results of the conventional PID and fuzzy PID no-load step response and sudden load simulation for the same PID initial value are shown in Fig 4. It can be seen from the figure that the fuzzy PID is faster than the conventional PID response speed in the no-load step, and the fuzzy PID is stronger than the conventional PID anti-load disturbance capability.

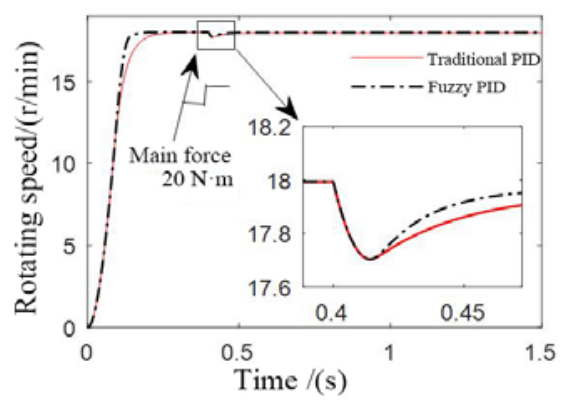

FIGURE IV. STEP AND SUDDEN LOAD SIMULATION RESULTS

\section{Training Trajectory Tracking Simulation Analysis}

In the actual rehabilitation training, the lower limb rehabilitation robot is controlled by the given target speed trajectory or the normal side limb movement speed trajectory. 
In order to more realistically reflect the control effect of the PID algorithm, the velocity trajectory after the active training is used as the tracking control target, and the Matlab-Adams joint simulation analysis is carried out. The simulation model is shown in Figure V. In the simulation, the discrete target velocity trajectory is imported into Simulink, and the target velocity trajectory is determined according to the linear interpolation of the hinge angle fed back by the Adams model. The sampling control period is $2 \mathrm{~ms}$, and the fuzzy PID parameters are as described above. The simulation results of the speed trajectory tracking without considering external disturbance such as friction are shown in Figure VI.

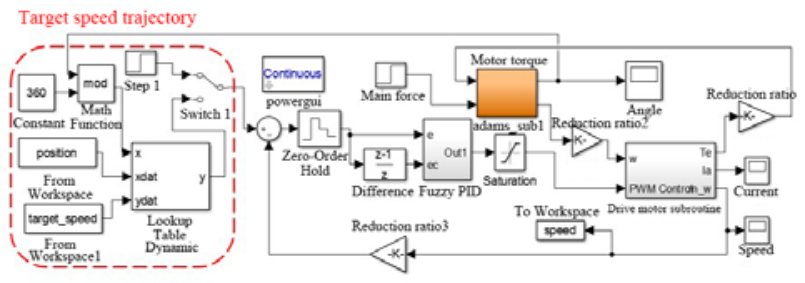

FIGURE V. MATLAB-ADAMS CO-SIMULATION MODEL

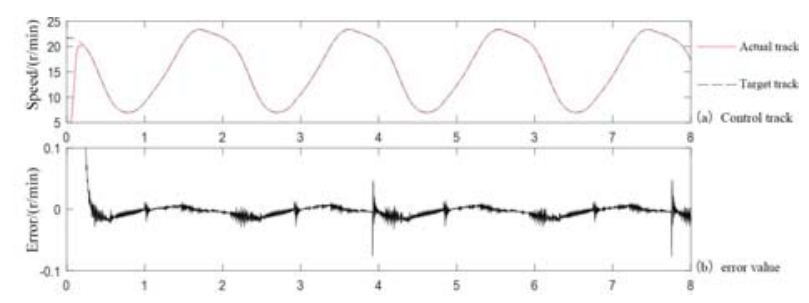

FIGURE VI. FUZZY PID SPEED TRAJECTORY SIMULATION RESULTS

It can be seen from Figure VI that the speed trajectory tracking error is within $\pm 0.1 \mathrm{r} / \mathrm{min}$, and the overall error is small. There is a slight turbulence in the error, which is caused by the discontinuity of the target speed. In the actual passive training, the target speed discontinuity should be avoided. At the beginning, the response time of $22 \mathrm{r} / \mathrm{min}$ speed is about $0.2 \mathrm{~s}$. There is no overshoot phenomenon, which can achieve smooth and fast response, and can realize passive training speed trajectory tracking.

\section{PAssive Training TRAJECTORY TRACKING EXPERIMENT}

In the simulation analysis, it is found that the expected speed trajectory in the training can not have a large mutation, otherwise it will have a greater impact on the patient. Therefore, in the actual rehabilitation training, a uniform acceleration transition phase is set before reaching the desired speed target. In order to test the actual control effect of fuzzy PID, learn from the experience of PID parameter adjustment in simulation, after adjusting the PID parameters and the scale factor of each parameter in the fuzzy control under no-load, the volunteers were subjected to passive rehabilitation training experiments with HT values of $10,30,50$ (HT value is the comprehensive state representation value of the human body, and the larger the HT value, the greater the patient's muscle strength). The lower limbs of the volunteer were driven by the machine to complete the movement. The experimental results are shown in Figure
VII(a). The calculated speed tracking error is shown in Figure VII(b).
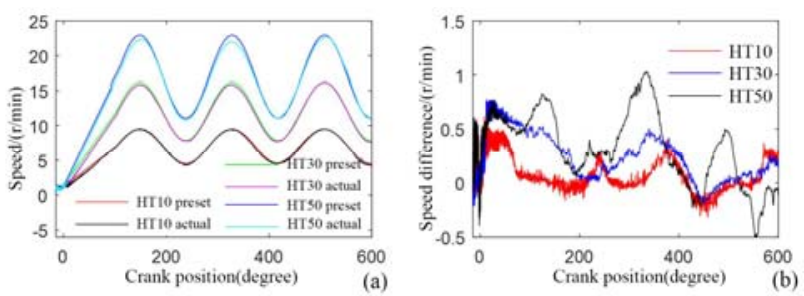

FIGURE VII. PASSIVE TRAINING SPEED-POSITION EXPERIMENTAL CURVE

From the experimental results, the dynamic error of the velocity trajectory is about $0.5 \mathrm{r} / \mathrm{min}$ and the steady-state error is about $0.22 \mathrm{r} / \mathrm{min}$ when the speed is low (such as the low-speed zero return phase). The dynamic error is relatively large. When following the desired motion trajectory, the dynamic tracking error is about $0.1 \mathrm{r} / \mathrm{min}$, and the steady-state error increases at the pole of the velocity trajectory. At HT50, the maximum is about $1 \mathrm{r} / \mathrm{min}$. The maximum steady-state error is not much different between different training intensities, the correlation between steady-state error and trajectory acceleration is large, and the correlation with training intensity is small. In general, the passive training motion control algorithm designed in this paper can meet the requirements of passive training.

\section{SUMMARY}

In this paper, by defining the fuzzy set of input and output variables and fuzzy logic, the fuzzy set, time series and membership degree of the input variable error value $e$ and the error change rate $e c$ are determined, and the defuzzification calculation of the output variable is completed. Therefore, the fuzzy following method is used to establish the speed following controller. Compared to traditional PID control, it responds faster to a given signal and is more resistant to disturbances. At the same time, the joint simulation model of control system and dynamics system is established based on Matlab and Adams software. The speed control effect of fuzzy PID is simulated and analyzed, and the feasibility of the algorithm is preliminarily verified.

\section{REFERENCES}

[1] Senanayake C, Emerging Robotics Devices for Therapeutic Rehabilitation of the Lower Extremity[C], IEEE International Conference on Advanced Intelligent Mechatronics. 2009, (7): 1142-1147.

[2] Peterson M D, Lukasik L, Muth T, et al, Recumbent Cross-Training Is a Feasible and Safe Mode of Physical Activity for Significantly Motor-Impaired Adults With Cerebral Palsy[J], Archives of Physical Medicine \& Rehabilitation, 2013, 94(2):401-7.

[3] Seraji H, Colbaugh R, Force tracking in impedance control [J], The International Journal of Robotics Research, 1997, 16(1): 97-117.

[4] Ferris D P, Sawicki G S, Domingo A, Powered lower limb orthoses for gait rehabilitation[J], Topics in spinal cord injury rehabilitation, 2005, 11(2): 34-49. 